\section{Ultra-Violet Solar Spectrum and Ozone in the Stratosphere}

ON June 26, July 7 and 31, we succeeded in sending up registering balloons into the stratosphere, to heights of 21,20 and $31 \mathrm{~km}$. respectively, with an automatic quartz spectrograph. The plate registering the spectra was moved every few minutes; simultaneously, two barographs and a thermograph recorded pressure and temperature on the photographic plate. In order to avoid the permanent position of the spectrograph direct towards the sun, a plate of magnesium oxide $(\mathrm{MgO})$ was fixed about 1.5 metres below the spectrograph and the latter was directed downwards to the MgO plate.

According to our present calculations, we find that at the first ascent, at a height of $21 \mathrm{~km}$., approximately 40 per cent of the whole layer of ozone was below the apparatus. At the third ascent, at a height of $31 \mathrm{~km}$., we calculate that 70 per cent of the ozone layer was below the apparatus. These results are remarkable because they confirm the new calculations of F. W. P. Gotz, A. R. Meetham and G. M. B. Dobson ${ }^{1}$, who deduce from the observations of the zenith sky light a height of the ozone layer which is considerably lower than previously supposed.

A more detailed report of the investigations will be published shortly in the Physikalische Zeitschrift. ERICH REGENER.

Physikalisches Institut der ViCTOR H. REGENER.

Technischen Hochschule, Stuttgart.

' Götz, Meetham and Dobson, Nature, 133, 281, Aug. 19, 1933. Proc. Roy. Soc., A, 145. 416; 1934.

\section{Nuclear Magnetic Moments and the Properties of the Neutron}

IN a recent letter to NATURE, S. Tolansky ${ }^{1}$ has discussed the "negative nuclear spins" of nuclei containing an odd number $N$ of neutrons and an even number $Z$ of protons. Nuclei of this group may have both positive and negative magnetic moments (that is, Landé's $g$-factor of these nuclei may be both positive and negative), whereas if $N$ is even and $Z$ odd, the magnetic moment is always positive.

Without entering into criticism of theoretical considerations raised by Dr. Tolansky (I can agree with Dr. Tolansky in the view that there may exist in Nature two kinds of neutrons-but scarcely of the structure proposed by him-but not in his further inferences), I should like to point out that his statement that "Tamm and Altschuler ... have attempted to explain the difficulty of negative spins by assuming that several neutrons [as opposed to a single neutron] can contribute to the spin properties" is not altogether correct. In the note cited by Tolansky ${ }^{2}$, Mr. Altschuler and I have shown that from the simple fact that the orbital motion of the neutron does not contribute to its magnetic moment, and from the generalised Landé formula for the $g$-factor, it follows immediately that the sign of the $g$-factor of a neutron, supposed to be moving in a spherically symmetrical field of the rest of the nucleus, depends on the state of the neutron, positive and negative signs being about equally frequent.
This result is independent of any assumption as to the relative direction of the magnetic moment and of the spin of a free neutron, and also holds when several neutrons contribute to the angular and the magnetic moments of the nucleus, as well as when a single neutron is concerned. Thus the facts considered by Dr. Tolansky may be easily explained without special assumptions. In fact, we made the assumption mentioned by Tolansky not to explain the 'negative spins', but only in order to obtain a consistent explanation of numerical values of the measured $g$-factors.

Teberda,

Ig. TAMM.

\section{Caucasus.}

July 24.

1 NATURE, 134, 26, July 7, 1934.

2.R. Acad. Sci. U.R.S.S., 1, $455 ; 1934$.

\section{Solubility of Gluten}

The tenacious mass obtained when the starch is washed from a dough of wheat flour is termed 'gluten', and is commonly held to contain two distinct proteins, glutenin and gliadin. Glutenin is defined in current textbooks as insoluble in all neutral solvents, but soluble in dilute acid and alkali. Gliadin is also defined as insoluble in neutral solvents, but is distinguishable from glutenin by its solubility in 60-80 per cent alcohol.

It has already been shown by Cook and Alsberg1 that gluten can be dispersed completely in 30 per cent urea solution, while only partial dispersion is obtained in concentrated solutions of the lyotropic salts, potassium iodide and potassium thiocyanate. Later studies ${ }^{2}$ of gluten dispersions in 30 per cent urea led to the conclusion that, although fractions resembling glutenin and gliadin could be obtained from such dispersions, there was no clear-cut distinction between the solubilities of these two proteins. Whole gluten was therefore used in subsequent investigations.

\begin{tabular}{|c|c|c|c|}
\hline $\begin{array}{c}\text { Sodium } \\
\text { salicylate. } \\
\text { Grams per } \\
\text { 100 c.c. } \\
\text { solution. }\end{array}$ & $\begin{array}{c}\text { Gluten nitrogen } \\
\text { extracted as } \\
\text { percentage total } \\
\text { gluten nitrogen. }\end{array}$ & $\begin{array}{c}\text { Urea. } \\
\text { Grams per } \\
\text { 100 c.c. } \\
\text { solution. }\end{array}$ & $\begin{array}{c}\text { Gluten nitrogen } \\
\text { extracted as } \\
\text { percentage total } \\
\text { gluten nitrogen. }\end{array}$ \\
\hline $7 \cdot 0$ & $92 \cdot 0$ & $18 \cdot 0$ & $85 \cdot 4$ \\
$8 \cdot 0$ & $99 \cdot 2$ & $21 \cdot 0$ & $94 \cdot 6$ \\
$9 \cdot 0$ & $99 \cdot 3$ & $24 \cdot 0$ & $99 \cdot 7$ \\
$10 \cdot 0$ & $99 \cdot 1$ & $27 \cdot 0$ & $99 \cdot 5$ \\
12.0 & 99.9 & & \\
\hline
\end{tabular}

Recently we have found that 10 and 12 per cent solutions of sodium salicylate would disperse gluten completely and more rapidly than solutions of the same substances at higher concentrations. Both urea and sodium salicylate are known to exert a denaturing action on albumins, and presumably the rate of denaturation increases with the concentration of reagent. This probably accounts for the lower rate of dispersion of gluten observed in sodium salicylate solutions of high concentration. An attempt was then made to determine more accurately the minimum concentration of urea and sodium salicylate required to disperse gluten. For this determination a tough, tenacious gluten obtained from a high-quality Canadian-grown wheat was employed, since concentrations capable of dispersing such material should disperse less tenacious glutens quite readily. The 